\title{
Korean Medication Algorithm Project for Bipolar Disorder 2018 (KMAP-BP 2018): Fourth Revision
}

\author{
Young Sup Woo", Won-Myong Bahk', Jung Goo Lee ${ }^{2,3}$, Jong-Hyun Jeong ${ }^{1}$, Moon-Doo Kim", InKi Sohn', \\ Se-Hoon Shim ${ }^{6}$, Duk-In Jon ${ }^{7}$, Jeong Seok Seo ${ }^{8}$, Kyung Joon Min ${ }^{9}$, Won Kim ${ }^{10}$, Hoo-Rim Song ${ }^{11}$, Bo-Hyun Yoon ${ }^{12}$ \\ ${ }^{1}$ Department of Psychiatry, College of Medicine, The Catholic University of Korea, Seoul, ${ }^{2}$ Department of Psychiatry, Inje University Haeundae \\ Paik Hospital, Inje University College of Medicine and Paik Institute for Clinical Research, Busan, ${ }^{3}$ Department of Health Science and \\ Technology, Graduate School of Inje University, Busan, ${ }^{4}$ Department of Psychiatry, Jeju National University Hospital, Jeju, ${ }^{5}$ Department of \\ Psychiatry, Keyo Hospital, Keyo Medical Foundation, Uiwang, ${ }^{6}$ Department of Psychiatry, Soonchunhyang University Cheonan Hospital, \\ Soonchunhyang University College of Medicine, Cheonan, ${ }^{7}$ Department of Psychiatry, Hallym University Sacred Heart Hospital, Hallym \\ University College of Medicine, Anyang, ${ }^{8}$ Department of Psychiatry, School of Medicine, Konkuk University, Chungju, ${ }^{9}$ Department of Psychiatry, \\ Chung-Ang University College of Medicine, Seoul, ${ }^{10}$ Department of Psychiatry, Inje University Seoul Paik Hospital, Inje University College \\ of Medicine, Seoul, ${ }^{11}$ Department of Psychiatry, Myongji Hospital, Goyang, ${ }^{12}$ Department of Psychiatry, Naju National Hospital, Naju, Korea
}

\begin{abstract}
Objective: The Korean Medication Algorithm Project for Bipolar Disorder (KMAP-BP) was first published in 2002 through an expert consensus of opinion, and updated in 2006, 2010, and 2014. This study constitutes the fourth revision of the KMAP-BP. Methods: A 50-item questionnaire was used to obtain the consensus of experts regarding pharmacological treatment strategies for various phases of adult bipolar disorder and six items for pediatric bipolar disorder. The review committee included 84 Korean psychiatrists and 43 child and adolescent psychiatry experts.

Results: The preferred first-step strategies for acute mania were the combination of a mood stabilizer (MS) and an atypical antipsychotic (AAP), MS monotherapy, and AAP monotherapy. A combination of a MS and an AAP, and AAP monotherapy were preferred for psychotic mania. The first-step strategies for mild to moderate bipolar depression were monotherapy with MS, AAP, or lamotrigine (LMT), and the combination of a MS and an AAP or LMT, or a combination of an AAP and LMT. The combination of two among a MS, AAP, and LMT were preferred for non-psychotic severe depression. A combination of a MS and an AAP or the combination of an AAP with an antidepressant or LMT were the first-line options for psychotic severe depression.

Conclusion: The recommendations of the KMAP-BP 2018 have changed from the previous version by reflecting recent developments in pharmacotherapy for bipolar disorder. KMAP-BP 2018 provides clinicians with a wealth of information regarding appropriate strategies for treating patients with bipolar disorder.
\end{abstract}

KEY WORDS: Bipolar disorder; Expert consensus; Drug therapy; Practice guideline.

\section{INTRODUCTION}

Received: May 9, 2018/ Revised: May 15, 2018

Accepted: May 16, 2018

Address for correspondence: Won-Myong Bahk, MD, PhD

Department of Psychiatry, Yeouido St. Mary's Hospital, College of

Medicine, The Catholic University of Korea, 10 63-ro,

Yeongdeungpo-gu, Seoul 07345, Korea

Tel: +82-2-3779-1051, Fax: +82-2-780-6577

E-mail: wmbahk@catholic.ac.kr

ORCID: https://orcid.org/0000-0002-0156-2510

Bo-Hyun Yoon, MD, PhD

Department of Psychiatry, Naju National Hospital, 1328-31,

Senam-ro, Sanpo-myeon, Naju 58213, Korea

Tel: +82-61-330-4152, Fax: +82-61-330-4150

E-mail: yoonbh@chollian.net

ORCID: https://orcid.org/0000-0002-3882-7930
Recent advances in psychopharmacology have led to rapid changes in the pharmacological treatment strategies for bipolar disorder. However, clinicians who treat bipolar disorders have limited time and resources to update their knowledge of the newest evidence on the efficacy, safety, and tolerability of the range of interventions available for the various and complex phases of bipolar disorder. Moreover, although several treatment algorithms and clinical practice guidelines have been published in many countries over the last two decades, ${ }^{1)}$ the

(ㄷ) This is an Open-Access article distributed under the terms of the Creative Commons Attribution Non-Commercial License (http://creativecommons.org/licenses/by-nc/4.0) which permits unrestricted non-commercial use, distribution, and reproduction in any medium, provided the original work is properly cited. 
treatment strategies used for clinical practice vary widely from country to country due to diverse health insurance policies, economic situations, and ethnicities. Therefore, several countries have initiated development of their own population-specific treatment guidelines. Physicians who treat mood disorders should search for guidelines, refer to information about new drugs and new clinical studies, and come to an understanding of the best treatment procedure for each case. However, it is anticipated that such efforts may be beyond the control of clinicians who are extremely busy. Therefore, the Korean Medication Algorithm for Bipolar Disorder has been developed, hoping to help physicians who treat bipolar disorders.

The Korean Medication Algorithm Project (KMAP) was a consensus-based guideline initiated in 2001, and the first set of guidelines, The Korean Medication Algorithm Project for Bipolar Disorder 2002 (KMAP-BP 2002), was published 1 year later. ${ }^{2)}$ The feasibility of the KMAP-BP 2002 was confirmed by additional studies showing that the algorithm could be successfully implemented in clinical settings in Korea. ${ }^{3-5)}$ The KMAP for Depressive Disorder (KMAP-DD) also has been published 2002, 2006, 2012, and 2017, 6,7 and KMAP-BP has been updated continually based on progress in psychopharmacology in 2006, ${ }^{8)} 2010,{ }^{9)}$ and $2014 .^{10)}$

Since publication of the KMAP-BP 2014, ${ }^{10)}$ various changes have been made with the introduction of fifth edition of the American Psychiatric Association Diagnostic and Statistical Manual for Mental Disorders (DSM-5), ${ }^{11)}$ and an abundance of novel information for treating bipolar disorder has become available. Therefore, the Korean College of Neuropsychopharmacology and the Korean Society for Affective Disorders have now created a fourth revision, KMAP-BP 2018, to reflect changes in expert opinion regarding the treatment of bipolar disorder, which we report here.

\section{METHODS}

The detailed methods regarding the constitution of the review committee, preparation of the questionnaire, data analyses, and development of the treatment guidelines and algorithms were similar to those in previous KMAP-BP studies. ${ }^{8-10)}$

\section{Review Committee}

Our review committee of experts recruited 84 Korean psychiatrists through invitation mail and e-mail to registered members of the Korean Society for Affective Disorders who had more than 15 years of clinical experience in the field of mood disorders. The committee members worked in a wide variety of clinical settings, including university hospitals $(n=57)$, general/psychiatric hospitals $(n=21)$, and private psychiatric clinics $(n=6)$. Responses were obtained from $61(72.6 \%)$ committee members. Additionally, 43 experts from the field of child/adolescent psychiatry were included on the review committee to aid with the development of the child/adolescent section. Of the 43 committee members, 30 (69.8\%) child/adolescent psychiatrists responded to the survey.

\section{Questionnaire}

The KMAP-BP 2018 is a set of expert consensus guidelines modeled after the 2002, 2006, 2010, and 2014 KMAP-BP assessments. The questionnaire used in the KMAP-BP 2018 is similar to the questions used to develop the KMAP-BP 2014, with some modifications. The major differences between KMAP-BP 2014 and KMAP-BP 2018 are as follows: The "treatment of mixed features" section was separated from the treatment of manic and depressive episodes based on changes in the DSM-5. We asked for an evaluation of the propriety use of available medications for the initial treatment strategy in one question (e.g., "please evaluate the propriety of the following medications as initial monotherapy for acute manic episode without psychotic features"). In the previous version, we had separated the questions by classifying the medications, such as atypical antipsychotics (AAPs), mood stabilizers (MSs), and antidepressants (e.g., "please evaluate the propriety of the following AAPs as initial monotherapy for acute manic episode without psychotic features"). In this questionnaire, we also asked about the propriety of each atypical antipsychotic agent as monotherapy and combination therapy with MSs, separately.

The KMAP-BP 2018 questionnaire for treatment of adult bipolar disorder has 50 main questions regarding important clinical situations, including 184 sub-items and 1,326 response options. In addition, six main questions, including 22 sub-items and 189 response options were included in the questionnaire for the treatment of child/adolescent bipolar disorder. 


\section{Rating Scale}

Of the 206 sub-items in the questionnaire for adult and child/adolescent bipolar disorder, 161 inquired about particular clinical cases and addressed the appropriateness of potential treatment options for these cases using a 9-point scale. This scale was based on the Expert Consensus Guideline Series: Medication Treatment of Bipolar Disorder 20002); a score of 9 indicated "extremely appropriate", a score of 7 or 8 indicated "usually appropriate" (first-line), a score of 4 to 6 indicated "equivocal appropriateness" (second-line), a score of 2 or 3 indicated "usually inappropriate" (a treatment you would rarely use), and a score of 1 indicated "extremely inappropriate" (a treatment you would never use). The reviewers were asked to consider ideal treatment options rather than those actually practiced and to choose " $q$ " if they had little experience or did not have available information for a particular question. Twenty-five questions were open-ended questions, and 30 were multiplechoice questions that ask the responder to select one or more treatment options.

\section{Medication Categories}

The medications were categorized as follows: typical antipsychotics (e.g., haloperidol, chlorpromazine, molindone, perphenazine, pimozide, etc.), AAPs (e.g., aripiprazole [ARI], olanzapine [OLZ], quetiapine [QUE], risperidone [RIS], and ziprasidone [ZIP]); other AAPs that were not approved for treating bipolar disorder by the Korean Ministry of Food and Drug Safety (e.g., amisulpride, blonanserin, paliperidone, and zotepine), MSs (e.g., lithium [LIT], valproic acid [VAL], and carbamazepine [CBZ]), and antidepressants (ADs; e.g., bupropion, desvenlafaxine, duloxetine, escitalopram, fluoxetine, milnacipran, mirtazapine, moclobemide, paroxetine, sertraline, tianeptine, tricyclic antidepressants, venlafaxine, and vortioxetine). Clozapine (CLZ) and lamotrigine (LMT) were not included in any of the categories and were treated as a category per se. Medications not available in Korea, e.g., asenapine, cariprazine, lurasidone, and agomelatine, were not included in this survey.

\section{Data Analysis and Development of the Treatment Guidelines and Algorithms}

The rating scores were assumed to be randomly distributed and the rating scores on the 9-point scale were divided into three groups (1-3, 4-6, and 7-9). A lack of a significant difference among groups was interpreted as reflecting a lack of consensus by the experts. The presence or absence of a consensus for each of the options/questions was determined using the chi-square test to identify significant differences. Then, the means and 95\% confidence intervals (Cls) of the scores were calculated and divided into three categories based on the lowest $\mathrm{Cl}$ score: $\geq 6.5$ for first-line/preferred treatments, $<6.5$ and $\geq 3.5$ for second-line/reasonable treatments, and $<3.5$ for third-line/inappropriate treatments. The first-line treatment options rated as 9 by $\geq 50 \%$ of the experts were defined as the treatment of choice (TOC); in other words, the most strongly recommended treatment. IBM SPSS Statistics software package (version 22.0; IBM Software Group, Somers, NY, USA) was used for the preference ranking and multiple response analyses.

The tables and algorithms for the guidelines were determined based on the preferred treatment strategies and medications identified by the present survey.

\section{Ethics}

The present study was conducted according to the Declaration of Helsinki, and the protocol was approved by the institutional review board (IRB) or ethics committee at each respective study site. The IRBs waived the requirement for informed consent for this survey. All respondents received a predetermined fee for their participation.

\section{RESULTS}

\section{Mania}

The combination of a MS and an AAP was the TOC and initial treatment strategy for mania, and AAP monotherapy was the first-line strategy for mania with or without psychotic features (Table 1). MS monotherapy was the first-line treatment strategy for non-psychotic mania but the second-line strategy for psychotic mania. VAL, LIT, OLZ, or QUE were recommended as the first-line monotherapy for non-psychotic mania, and ARI or RIS the second-line. OLZ was the TOC for psychotic mania, and QUE, RIS, or ARI were recommended as the first-line. OLZ or QUE were recommended as the TOC when AAPs were combined with LIT for non-psychotic mania, and RIS or ARI were the first-line. The first-line recommendations in cases of a combination with VAL were 
Table 1. Preferred initial treatment strategies and medications for acute mania and hypomania

\begin{tabular}{lll}
\hline & \multicolumn{1}{c}{ First-line strategy } & \multicolumn{1}{c}{ First-line medication } \\
\hline Mania without psychotic features & MS+AAP* & Monotherapy: VAL, LIT, OLZ, QUE \\
& MS/AAP monotherapy & Combination therapy: LIT+OLZ*/QUE*/RIS/ARI, VAL+QUE/OLZ/ARI/RIS \\
Mania with psychotic features & MS+AAP* & Monotherapy: OLZ*, QUE, RIS, ARI \\
& AAP monotherapy & Combination therapy: LIT+OLZ*/QUE*/RIS/ARI, VAL+OLZ*/QUE/RIS/ARI \\
Hypomania & MS monotherapy* & Monotherapy: VAL*, QUE, LIT, ARI, OLZ \\
& AAP monotherapy & \\
\hline
\end{tabular}

MS, mood stabilizers; AAP, atypical antipsychotics; VAL, valproate; LIT, lithium; OLZ, olanzapine; QUE, quetiapine; RIS, risperidone; ARI, aripiprazole.

*Treatment of choice.

QUE, OLZ, ARI, or RIS. LIT with OLZ or QUE, and VAL with OLZ were the TOC for the combined treatment of psychotic mania. LIT with RIS or ARI and VAL with QUE, RIS, or ARI were recommended as the first-line treatment.

In cases where patients responded poorly to initial monotherapy with one of the MSs, augmentation with an AAP was the TOC for both a partial response and non-response as the second-step treatment strategy. If the patient did not show a partial response to AAP monotherapy, adding a MS was the TOC for both types of mania. Adding another AAP was recommended as the first-line treatment for psychotic mania and the second-line for non-psychotic mania. Adding a MS was the TOC for non-psychotic mania and the first-line for psychotic mania in patients who did not respond to AAP monotherapy. Switching an AAP to another AAP or adding another AAP were first-line treatment strategies for a non-response to psychotic mania and second-line for non-responding non-psychotic mania.

In cases where patients partially responded to the initial combined treatment with a MS and an AAP, the recommended first-line strategies were adding another AAP or MS, or switching the AAP to another AAP for both types of mania. Switching the MS to another MS was also recommended as the first-line treatment for mania without psychotic features. When patients did not respond to a MS and AAP combined treatment, switching the AAP to another AAP or adding another AAP were first-line treatments for both types of mania. Switching the MS to another MS or adding another MS were recommended as first-line treatments only for non-responders with nonpsychotic mania.

When second-step combined treatment with LIT, VAL, and one AAP failed, switching the AAP to another AAP or adding another AAP were recommended as first-line treatments for mania with or without psychotic features. Switching LIT or VAL to another AAP was the first-line treatment for psychotic mania and the second-line treatment for non-psychotic mania. Switching LIT or VAL to CBZ and use of CLZ were second-line treatments for manic patients with psychotic features who did not respond to the combination of LIT, VAL, and an AAP. If a patient showed an insufficient response to step two treatment with two AAPs and the LIT or VAL combination, switching one of the pre-existing AAPs to another AAP was recommended as the first-line strategy for both types of mania. Switching the MS to another MS, switching the AAP to another MS, and adding another MS were also recommended as first-line treatments for non-psychotic mania. Adding another AAP or MS, switching the MS to another MS, and using CLZ were recommended as the second-line strategies for psychotic mania. Figure 1 presents the algorithm for treating mania.

\section{Hypomania}

The recommended TOC for hypomania was MS monotherapy, and the first-line treatment was AAP monotherapy (Table 1). The preferred medications were $\mathrm{VAL}$, QUE, LIT, ARI, and OLZ. Among them, VAL was the TOC. In cases where patients showed partial or non-response to initial MS monotherapy, switching the MS to another MS or an AAP, and augmentation with an AAP were recommended as the first-line treatment. If patients partially responded to AAP monotherapy, adding a MS was the TOC and adding an AAP was the first-line strategy. In cases of a non-response to initial AAP monotherapy, switching the AAP to another AAP or a MS, and adding a MS were recommended as the first-line treatment. The first-line treatment options for an insufficient response to the combination of a MS and an AAP was switching the MS to another 

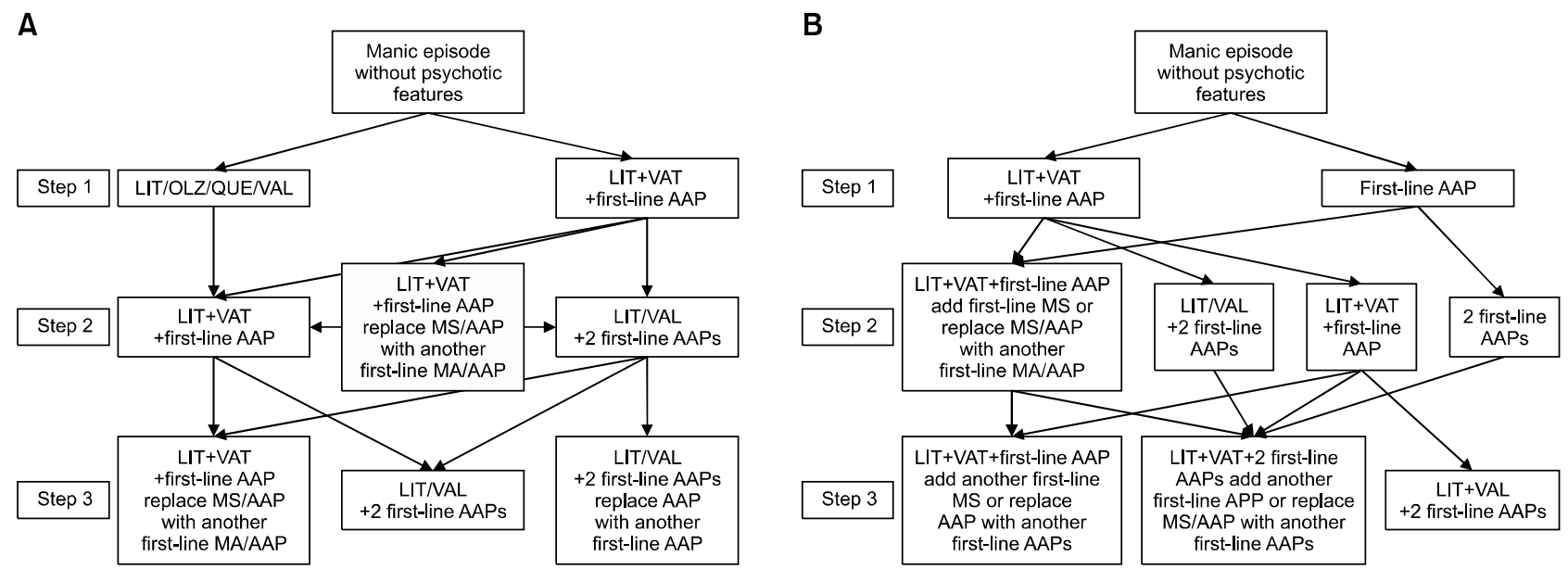

Fig. 1. The Korean Medication Algorithm for Bipolar Disorder 2018: Manic episode. (A) Manic episode without psychotic features. (B) Manic episode with psychotic features. Electroconvulsive therapy can be applied based on the clinician's decision at any time. First-line AAPs include aripiprazole, OLZ, QUE, and risperidone. First-line MSs include lithium and valproate.

LIT, lithium; OLZ, olanzapine; QUE, quetiapine; VAL, valproate; AAP, atypical antipsychotics; MS, mood stabilizers.

Table 2. Preferred initial treatment strategies and medications for acute bipolar depression

\begin{tabular}{lcll}
\hline & First-line strategy & \multicolumn{1}{c}{ First-line MS } & \multicolumn{1}{c}{ First-line AAP } \\
\hline Mild to moderate depression & MS/LMT/AAP monotherapy & Monotherapy: LIT, VAL, LMT & Monotherapy: ARI, QUE \\
& MS+AAP & Combination therapy: LMT*, LIT, VAL & Combination therapy: ARI, OLZ, QUE \\
MS+LMT & & \\
Non-psychotic severe depression & AAP+LMT & & \\
& MS+AAP & & \\
Psychotic depression & MAP+LMT & Monotherapy: QUE, OLZ, ARI \\
& MS+AAP* & Combination therapy: QUE*, OLZ, ARI \\
\hline
\end{tabular}

MS, mood stabilizers; AAP, atypical antipsychotics; LMT, lamotrigine; LIT, lithium; VAL, valproate; ARI, aripiprazole; QUE, quetiapine; OLZ, olanzapine.

*Treatment of choice.

MS, switching the AAP to another AAP, or adding another AAP. Adding another MS was also recommended as the first-line strategy for partial responders to the combination of a MS and an AAP.

\section{Bipolar Depression}

Monotherapy with a MS, AAP, or LMT, a combination of a MS and an AAP or LMT, or combination of an AAP and LMT were the first-line recommendations as the initial treatment strategy for mild to moderate depression (Table 2). The first-line recommendation for initial treatment of non-psychotic severe depression was the combination of two among a MS, an AAP, and LMT. The combination of a MS and an AAP was the TOC for patients with psychotic de- pression, and the combination of an AAP with an AD or LMT was also recommended as the first-line treatment (Table 2). The TOC was LMT for non-psychotic depression, and QUE for psychotic depression. The first-line recommendations included LIT, VAL, LMT, ARI, or QUE for monotherapy, LIT, VAL, ARI, OLZ, or QUE for combined therapy for non-psychotic depression and QUE, OLZ or ARI for monotherapy, and OLZ or ARI for combined therapy for psychotic depression. Escitalopram, bupropion, and sertraline were preferred when the use of an AD was necessary.

When the response to the initial treatment strategy was insufficient, adding an AAP or LMT was the first-line second step treatment for partial or non-responders with mild to moderate depression to initial MS monotherapy. In ad- 
dition, adding LMT was the TOC for partial responders. In cases of a partial or non-response to initial combined treatment with a MS and an AAP, the first-line recommendations were adding LMT or an AAP, or switching the AAP to another AAP. Switching the MS to another MS or LMT was the first-line treatment for non-responders. If the initial treatment with the combination of a MS and an AAP showed an insufficient response, adding LMT or an AAP, or switching the AAP to another AAP were recommended as the first-line treatment for both partial and nonresponders. Adding $\mathrm{AD}$ or switching the $\mathrm{AAP}$ or $\mathrm{MS}$ to LMT were also recommended as the first-line treatment for non-responders. If the initial strategy was the combination of a MS and an AD, switching the MS to another MS or LMT, or adding an AAP or LMT were first-line treatments for non-responders, and adding an AAP or LMT was the first-line treatment for partial responders. When the initial treatment with the combination of an AAP and LMT failed, adding a MS or an AAP was the first-line recommendation for both partial and non-responders. When the combination of a MS and an AD failed, adding an MS, $\mathrm{LMT}$, or an AAP, or switching the AAP to another AAP were recommended as first-line treatments.

Use of CLZ, buspirone, thyroid hormone, or a psychostimulant were recommended as second-line treatment options when second-step treatment failed to produce a response. Figure 2 presents the algorithm for treating bipolar depression.

\section{Mixed Features}

In KMAP-BP 2018, mixed features were subdivided into three types of mixed features with predominant manic symptoms (mania with mixed features/mixed mania), mixed features with predominant depressive symptoms (depression with mixed features/mixed depression), and mixed features without predominance, which were similar in the severity of manic and depressive symptoms. The initial treatment strategy for mania with mixed fea-

Table 3. Preferred initial treatment strategies for mixed features

\begin{tabular}{ll}
\hline & First-line strategy \\
\hline Mixed mania & MS+AAP* \\
\multirow{3}{*}{ Mixed depression } & AAP monotherapy \\
& MS+LMT \\
Mixed features without predominance & MS+AAP \\
& AAP+LMT \\
& AAP monotherapy \\
\hline
\end{tabular}

MS, mood stabilizers; AAP, atypical antipsychotics; LMT, lamotrigine. *Treatment of choice.

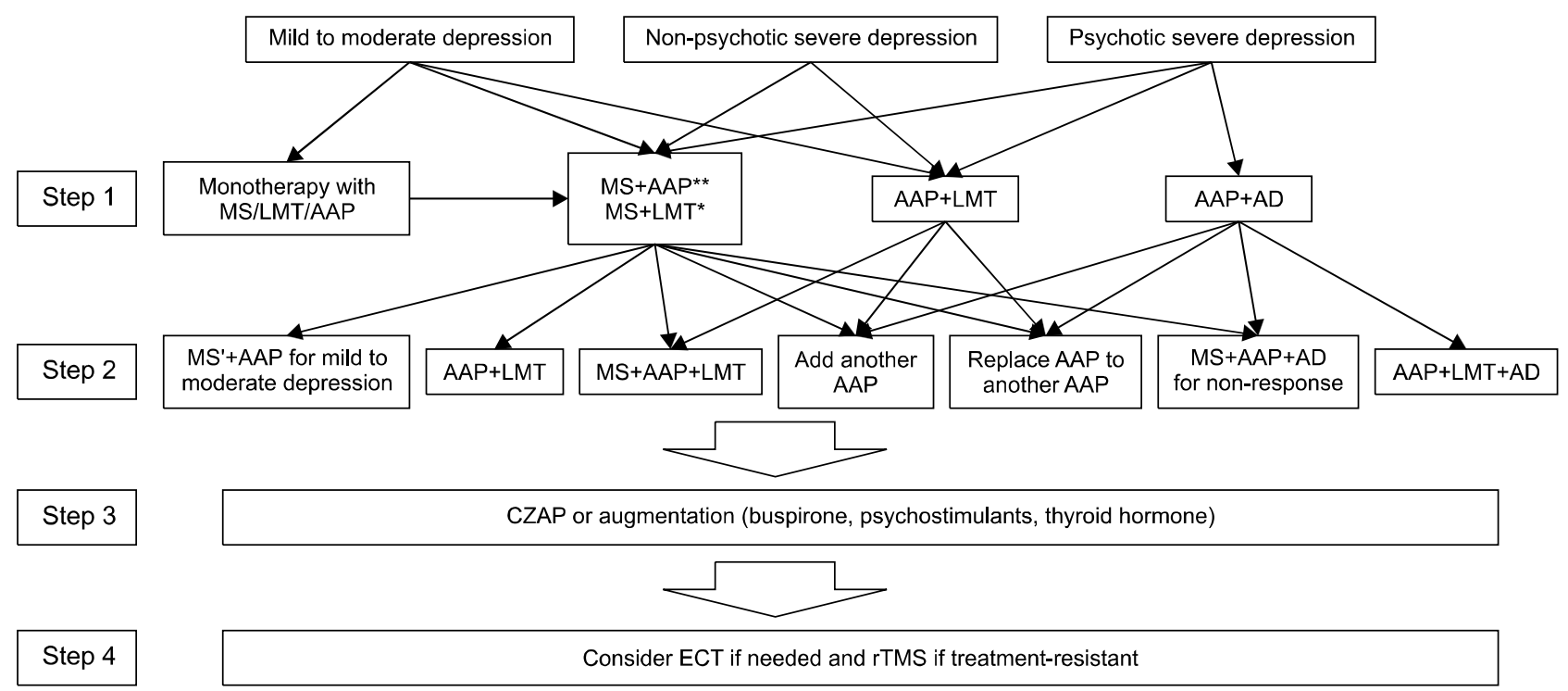

Fig. 2. The Korean Medication Algorithm for Bipolar Disorder 2018 (KMAP-BP 2018): Depressive episode. Electroconvulsive therapy can be applied based on a clinician's decision at any time. MS' means to replace MS with another MS. *Step 1 treatment strategy only for non-psychotic depression. **Treatment of choice.

MS, mood stabilizers; LMT, lamotrigine; AAP, atypical antipsychotics; AD, antidepressants; CZAP, clozapine; ECT, electroconvulsive therapy; rTMS, repetitive transcranial magnetic stimulation. 
tures and mixed features without predominance were identical. The TOC was the combination of a MS and an AAP. Monotherapy with an AAP was also the first-line treatment (Table 3). The preferred medication as monotherapy was VAL as the TOC, and LIT, ARI, OLZ, or QUE as first-line treatments for mixed mania. All of these medications including VAL were graded as the first-line choice for mixed features without predominance. The combination of two among a MS, an AAP, and LMT was the first-line recommendation for depression with mixed features, and LIT, VAL, LMT, ARI, OLZ, or QUE were recommended as first-line medications for monotherapy.

If patients with mixed mania insufficiently responded to initial treatment with MS monotherapy, adding an AAP (TOC) or another MS (first-line) was recommended. In cases of an insufficient response to initial treatment with AAP monotherapy, adding a MS was the TOC and switching to, or adding another, AAP was the first-line recommendation. When the combination of a MS and an AAP failed, switching the MS to another MS or the AAP to another AAP, or adding a MS or AAP were recommended first-line treatments.

Switching to, or adding another, first-line medication was recommended in cases where the initial treatment showed an insufficient response in patients with mixed features without predominance. If monotherapy with a MS failed, adding an AAP was the TOC and adding another MS was the first-line. When AAP monotherapy failed, adding a MS was the TOC. If the first-line strategy for monotherapy with LMT did not show a sufficient response, adding a MS or an AAP was the first-line. Adding another MS or AAP, and switching the MS to another MS or the AAP to another AAP were recommended as first-line treatments. After the combination of MS and LMT as the initial strategy, adding an AAP was the TOC and switching the MS or LMT to AAP was the first-line option as the next step strategy. When the combination of AAP and LMT treatment was unsuccessful, adding a MS or another AAP was recommended. Switching AD to MS or adding an AAP for the unsuccessful initial combination of MS and $A D$, and switching $A D$ to $A A P$ or adding a MS for an unsuccessful initial combination of $A A P$ and $A D$ were recommended as the next step.

The recommended second-step strategy varied from the initial treatment strategy when patients with depression and mixed features did not show a sufficient response. In cases of an insufficient response to monotherapy, adding an AAP or LMT for MS monotherapy, adding an AAP, a MS, or LMT for AAP monotherapy, and adding a MS or an AAP for LMT monotherapy were used. If patients poorly responded to the combination of a MS and an AAP, switching the AAP to another AAP or adding another MS, AAP, or LMT were recommended as first-line treatments. Switching the MS to an AAP or adding an AAP were recommended in cases of a poor response to the combination of MS and LMT. If the combination of an AAP and LMT failed, then adding a MS or an AAP, or switching the AAP to another AAP were first-line treatments. When $A D$ was used in combination with a MS as the first-step and failed, switching the AD to an AAP or adding an AAP or LMT were recommended. If the first step was the combination of AD and an AAP, then adding a MS or LMT should be considered as the second-step strategy.

\section{Rapid Cycling}

The first-line treatment for rapid cycling was the combination of a MS and an AAP for both manic and depressive phase rapid cycling. In particular, this combination was the TOC for the manic phase. Additionally, a MS with LMT therapy and an AAP with LMT combinations were first-line treatments during the depressive phase. The first-line medications for the manic, depressive, mixed, and hypomanic phases were VAL, QUE, OLZ, and ARI, respectively. LIT was the first-line medication for depressive and hypomanic phases and LMT was the first-line medication for treatment of the depressive phase.

Table 4. Preferred treatment strategies for maintenance

\begin{tabular}{ll}
\hline & First-line strategy \\
\hline Preventing manic relapse & MS+AAP \\
& MS monotherapy \\
Preventing depressive relapse & AAP monotherapy \\
& MS+AAP \\
& MS+LMT \\
& AAP+LMT \\
MS monotherapy \\
LMT monotherapy \\
AAP monotherapy \\
MS+AAP+LMT \\
\hline
\end{tabular}

MS, mood stabilizers; AAP, atypical antipsychotics; LMT, lamotrigine. 


\section{Maintenance Therapy}

The first-line strategy to prevent a manic episode included monotherapy with a MS or AAP, and combination therapy with a MS and an AAP (Table 4). The preferred AAP was ARI, QUE, or OLZ for both monotherapy and in combination with a MS. In particular, ARI was the TOC for the combination with a MS. In cases when acute treatment for manic symptoms with a MS and AAP combination was successful, $78.0 \%$ of experts recommended not to discontinue the AAP during maintenance therapy. The mean recommended duration to maintain AAP by experts who chose to discontinue AAP was 12.3 weeks after reaching remission.

Monotherapy with a MS, an AAP, or LMT, a combination of two of these three medications, and a triple combination of these three medications were all recommended as first-line maintenance therapy to prevent a depressive episode (Table 4). When the use of AD was constrained during the maintenance phase, bupropion, escitalopram, or sertraline should be considered. In cases when acute treatment for depression with AD combined with a MS or AAP was successful, the experts recommended discontinuing the AD 7.0 weeks after reaching remission for mild to moderate depression, 9.2 weeks for non-psychotic severe depression, and 8.4 weeks for psychotic depression. In total, $23 \%$ of the experts maintained AD as long as possible for psychotic depression, and the proportion of experts who chose to maintain AD was $14.8 \%$ for non-psychotic severe depression and $4.9 \%$ for mild to moderate depression.

When remission was attained from a mixed state by polypharmacy, the proportion of experts recommending not to discontinue AAP for mixed mania was $83.6 \%$, $80.3 \%$ for mixed depression, and $78.7 \%$ for mixed features without predominance. About $70.5 \%$ of experts recommended not discontinuing a MS during the maintenance phase for mixed mania, $62.3 \%$ for mixed depression, and $68.9 \%$ for mixed features without predominance. The recommended duration for using AD after a mixed state was 4.8 weeks for mixed mania and 8.5 weeks for mixed depression.

Monotherapy with a MS or AAP, or a combination of a $\mathrm{MS}$ and an AAP were also recommended as maintenance therapy for bipolar II disorder. LIT and VAL among MSs, and ARI, QUE, and OLZ among AAPs were preferred for maintenance therapy. LMT was also recommended as the first-line treatment for preventing a depressive episode in bipolar II disorder.

\section{Pharmacotherapy Considering Safety, Tolerability, and Medical Comorbidities}

When the patient's weight increased significantly due to pharmacotherapy, the primary recommendations included behavioral and diet modifications and switching to another medication with a low risk of weight gain. If additional medications were needed to counteract the weight gain, metformin, topiramate, orlistat, and bupropion should be considered. LMT, ARI, and ZIP were the first-line treatment strategies for patients with concerns for weight gain. In patients with signs or symptoms of hyperprolactinemia, including amenorrhea or galactorrhea, switching to an AAP with a low risk for hyperprolactinemia was the first-line treatment option, and a dose reduction of the current medication was the second-line option. If benign skin rashes appeared during LMT treatment, reducing the dose and close monitoring were first-line treatment options.

$\mathrm{ARI}$ and ZIP were the first-line recommendations for patients comorbid with metabolic disorders when considering both efficacy and safety/tolerability. ARI was also the first-line treatment for patients comorbid with cardiovascular or renal comorbidities. LIT and ARI were recommended for patients with hepatic comorbidities, and VAL and ARI were recommended for cerebrovascular comorbidities.

\section{Pharmacological Management of Special Populations}

No first-line recommendation was made for women of child-bearing age. ARI was the most preferred medication, but it was graded as second-line. VAL and CBZ were the third-line, which means inappropriate and should be avoided. If pharmacological treatment was needed to treat bipolar disorder in women during the first trimester of pregnancy, RIS, ZIP, other AAPs, and AD were preferred as the second-line, and there was no consensus about using ARI, OLZ, and QUE. There was no first-line recommendation for women during the second and third trimesters of pregnancy; LIT, VAL and CBZ were regarded as third-line options. ZIP, other AAPs, and AD were recommended as the second-line, and $L I T, V A L$, $\mathrm{CBZ}$, and LMT were not recommended for lactating women. 
Table 5. Preferred initial treatment strategies and medications for pediatric and geriatric bipolar patients

\begin{tabular}{|c|c|c|c|}
\hline & First-line strategy & First-line MS & First-line AAP \\
\hline \multirow[t]{2}{*}{ Mania in elderly } & AAP monotherapy & VAL, LIT & ARI, QUE, OLZ \\
\hline & MS monotherapy & & \\
\hline \multirow[t]{5}{*}{ Depression in elderly } & AAP monotherapy & LMT, VAL, LIT & ARI*, QUE, OLZ \\
\hline & MS monotherapy & & \\
\hline & MS+AAP & & \\
\hline & AAP+LMT & & \\
\hline & MS+LMT & & \\
\hline \multirow[t]{2}{*}{ Mania in children } & $\mathrm{MS}+\mathrm{AAP}$ & No first-line recommendation & RIS, ARI \\
\hline & AAP monotherapy & & \\
\hline Depression in children & No first-line recommendation & No first-line recommendation & ARI \\
\hline \multirow[t]{2}{*}{ Mania in adolescents } & MS+AAP & VAL, LIT & RIS*, ARI, QUE \\
\hline & AAP monotherapy & & \\
\hline Depression in adolescents & $\mathrm{AAP}+\mathrm{LMT}$ & VAL & ARI*, RIS, QUE \\
\hline
\end{tabular}

MS, mood stabilizers; AAP, atypical antipsychotics; VAL, valproate; LIT, lithium; ARI, aripiprazole; QUE, quetiapine; OLZ, olanzapine; LMT, lamotrigine; RIS, risperidone.

*Treatment of choice.

Monotherapy with AAP or a MS were recommended as first-line treatment strategies for geriatric bipolar patients with acute mania (Table 5). VAL and LIT among MSs and ARI, QUE and OLZ among AAPs were preferred to treat manic symptoms. In cases of bipolar depression, monotherapy with an AAP or a MS, and a combination of two among MS, AAP, and LMT were the first-line treatment strategies. The recommended first-line MSs for bipolar depression were $\mathrm{LMT}, \mathrm{VAL}$, and $\mathrm{LIT}$, and the recommended first-line AAPs were ARI, QUE, and OLZ. In particular, ARI was the TOC for treatment of bipolar depression in the elderly. The preferred ADs were escitalopram, sertraline, and bupropion. In cases where dementia was comorbid with bipolar disorder, VAL and ARI were recommended as the first-line treatment options.

The first-line treatment strategies for manic episodes in children (age $\leq 12$ years) and adolescents (age 13-18 years) were the combination of a MS and an AAP, and AAP monotherapy (Table 5). Among AAPs, ARI and RIS were the first-line options for children and adolescents, and QUE was also recommended as the first-line for adolescents. RIS was the TOC for adolescents with mania. Among MSs, VAL and LIT were the first-line recommendations for adolescents, but there was no first-line recommendation among MSs for children; VAL was the most preferred but graded as the second-line treatment for children with mania. The combination of an AAP and LMT was recommended for treating bipolar depression in adolescents, but there was no consensus regarding the first-line treatment strategy in children. In cases where pharmacological treatment was necessary, ARI was recommended as the first-line treatment for children and the TOC for adolescents. RIS, QUE, and VAL were also included as first-line options for adolescent bipolar depression. No consensus regarding a first-line treatment strategy for children or adolescents with depressive episodes and at high risk for bipolar disorder (e.g., family history of bipolar disorder) was reached.

\section{DISCUSSION}

\section{Mania and Hypomania}

The most remarkable changes from the previous version, KMAP-BP 2014, ${ }^{10)}$ for treatment of mania/hypomania were increased preference for AAPs in the treatment strategy, and changes in preference for individual AAPs. In the previous version, AAP monotherapy was the first-line treatment only for psychotic mania but graded as the second-line for non-psychotic mania. However, it is recommended as the first-line for both psychotic and non-psychotic mania in KMAP-BP 2018. Moreover, among AAPs, OLZ and QUE are the first-line options for both AAP monotherapy and combination therapy with a MS in patients with non-psychotic mania, but ARI and RIS are recommended as the first-line only for combination therapy with a MS in this version.

AAP monotherapy has been recommended as the first-line treatment for non-psychotic mania in evi- 
dence-based guidelines, ${ }^{13,14)}$ but it was recommended as the second-line in KMAP-BP 2014. This might reflect the fact that Korean experts were doubtful of the clinical effectiveness of AAP monotherapy and emphasized the superiority of combination therapy over monotherapy in terms of efficacy for mania based on the results from clinical trials and meta-analyses. ${ }^{15-19)}$ However, changes in the level of the recommendations for AAP monotherapy from second-line to first-line in KMAP-BP 2018 might reflect the increased concerns for adherence and burden of adverse events that negatively affect the combination treatment. $^{20,21)}$ The equivalent recommendation level for AAP monotherapy and the combination of an AAP and a MS was supported by a recent meta-analysis suggesting that the efficacy advantages of antipsychotics and MS combination versus monotherapy should be balanced with its greater burden of adverse events. ${ }^{22)}$

The difference in the recommendation grade among AAPs for monotherapy in the KMAP-BP 2018, e.g., OLZ and QUE as the first-line and ARI and RIS as the second-line, is not easy to explain. The rank order of anti-manic efficacy among AAPs appeared to be RIS, OLZ, QTP, ARI, and ZIP in a meta-analysis of comparative efficacy and acceptability of anti-manic drugs for acute mania. ${ }^{23)}$ This meta-analysis also suggested the superiority of RIS and OLZ over other AAPs in terms of efficacy. However, OLZ was not recommended as the first-line treatment for mania in recent evidence-based guidelines due to safety and tolerability concerns, although it has level 1 evidence for efficacy. ${ }^{24,25)}$ In this respect, the preference for OLZ could be interpreted as a residual of the propensity of Korean experts to emphasize efficacy over safety and tolerability. Furthermore, the grading of the recommendation for OLZ and QUE into the first-line, but RIS into the second-line, may be because the Korean experts were concerned about the relapse-preventing effect of medications when treating patients with acute mania. QUE and OLZ monotherapy are known to reduce the overall risk of relapse, ${ }^{24,26)}$ but overall efficacy of RIS to prevent any relapse remains controversial. ${ }^{24,27)}$

Another change was that monotherapy with MS or AAP was not recommended as the first-line strategy in the second-step when initial monotherapy with a MS or AAP had failed. AAP monotherapy was the first-line recommendation in the KMAP-BP 2014 for cases of non-response to initial monotherapy with MS or AAP. These re- sults from the KMAP-BP 2018 also reflect the above-mentioned evidence for the superiority of combined therapy over monotherapy. ${ }^{19)}$

\section{Bipolar Depression}

As a whole, the number of first-line recommendations was higher for treating depression than for mania. It could be interpreted as a lack of conclusive treatment strategies for treating bipolar depression, and this explains why clinicians often have difficulties treating patients with bipolar depression. When compared to the KMAP-BP 2014, the recommendation level for LMT and AAPs, particularly QUE and ARI, was upgraded and was included in the first-line strategy in various combinations with other medications for treating various clinical situations in the depressive phase.

Korean experts recommended LMT as the first-line option for the initial treatment strategy along with MSs and AAPs, although the efficacy of LMT for acute bipolar depression has not been established. ${ }^{28)}$ However, researchers indicated the methodological flaws in double blind trials reporting negative results, ${ }^{29)}$ and the superiority of LMT over placebo was reported in a meta-analysis as a monotherapy and augmentation strategy. ${ }^{30)}$ Moreover, recent evidence-based guidelines recommend the use of LMT in patients with bipolar depression. ${ }^{24,31,32)}$

Notably, the level of recommendations for ADs decreased, whereas the level increased for AAPs. The combination of a MS and an AD was recommended as the first-line treatment in KMAP-BP 2014, but the recommendation for this combination is downgraded to second-line in this revision. Evidence does not support the use of ADs in patients with bipolar depression ${ }^{33,34)}$ and it remains controversial. ${ }^{35)}$ The changes in the recommendation level for AD use in the KMAP-BP 2018 might reflect that all evidence-based guidelines consistently recommend limited use of ADs adjunctively with anti-manic agents in cases when ADs are needed. ${ }^{36)}$

Among AAPs, the efficacy of QUE for bipolar depression has been well established. ${ }^{37,38)}$ However, the efficacy of ARI for bipolar depression, which was recommended as the first-line option as monotherapy, and as adjunctive therapy, for psychotic and non-psychotic bipolar depression in KMAP-BP 2018, was not supported by evidence available thus far. ${ }^{39)}$ Thus, ARI was recommended as the third-line, as adjunctive therapy, and not recommended 
as monotherapy with level 1 negative evidence in the Canadian Network for Mood and Anxiety Treatments and International Society for Bipolar Disorders 2018 guidelines for managing patients with bipolar disorder, ${ }^{24)}$ and The International College of Neuropsychopharmacology Treatment Guidelines for Bipolar Disorder in Adults recommended ARI monotherapy as the third step. ${ }^{25)}$ However, some researchers have suggested the effectiveness of ARI monotherapy for treating acute depression. In a pooled, post-hoc analysis from two randomized, controlled trials that reported negative results, improvement in essential depressive symptoms measured with the six-item Montgomery-Åsberg Depression Rating Scale subscale score occurred with ARI monotherapy in subjects with more severe bipolar depression than those with less severe symptoms. ${ }^{40)}$ Moreover, a meta-analysis of data from the two negative studies ${ }^{41)}$ also suggested the usefulness of ARI monotherapy for bipolar depression. Furthermore, ARI has been increasingly prescribed for patients with bipolar depression at a continuously decreasing dosage. In a retrospective study with psychiatric inpatients, ${ }^{42)}$ the proportion of patients with bipolar depression who were prescribed ARI increased from 1.4\% (2004-2006) to $8.5 \%$ (2011-2014), but the prescribed mean initial and maximum dose was 15 and $30 \mathrm{mg} /$ day, respectively in 2004 to 2006 and was 6.3 and 16.8 $\mathrm{mg} /$ day, respectively in 2011 to 2014. The preference for, and high usage of, ARI for treating bipolar depression in Korea could be based on evidence supporting the efficacy of ARI for treating bipolar depression or result from the unavailability of certain medications including lurasidone, which was recommended as the first-line in other evidence-based guidelines in Korea. However, as the expert opinions and above studies suggest, the possibility that ARI could be effective for treating bipolar depression in a subset of patients at a relatively low dose should not be overlooked.

\section{Mixed Features}

Polypharmacy, which much evidence supports, ${ }^{43)}$ is highly recommended for treating patients with mixed features. Various combinations with AAPs and MSs were recommended for mixed mania and mixed features without predominance at the first-step and following steps, with the exception that AAP monotherapy was recommended as the first-line for mixed mania and mixed fea- tures without predominance at the first-step. Various combinations of AAPs, MSs, and LMTs were recommended for mixed depression. The preferred medications were VAL, LIT, ARI, OLZ, and QUE for all three types of mixed features, and LMT was also included for mixed depression.

These results were similar with the KMAP-BP 2014, ${ }^{10)}$ but there are some inconsistencies based on clinical trial evidence. CBZ and ZIP were not recommended for any type of mixed state in the KMAP-BP 2018, although the efficacy of $\mathrm{CBZ}^{44)}$ and $\mathrm{ZIP}^{45)}$ against manic and depressive symptoms in mixed patients has been reported. In contrast, LIT was recommended as the first-line for all three types of mixed states, although the presence of a mixed state could be a predictor of a poor response. ${ }^{46)}$ These findings suggest that the Korean experts set a high value on the versatility of medications and were concerned with the adherence problem when treating bipolar disorder. The preferred medications, including VAL, LIT, ARI, OLZ, and QUE are recommended as first-line monotherapy or as combination therapy for mania and depression in the KMAP-BP 2018. However, while CBZ and ZIP have proven efficacy for mania and mixed states, ${ }^{44,45,47,48)}$ their efficacy for bipolar depression has not been supported by clinical trials. ${ }^{49,50)}$ The use of medication that could be effective for mixed states but less effective for a more durable depressive state may not be preferred in real clinical situations. ${ }^{51)}$ Moreover, frequent intolerance to $\mathrm{CBZ},{ }^{52,53)}$ and relatively weak anti-manic efficacy ${ }^{23)}$ and frequent dosing requirements for ZIP could also negatively affect the preference.

\section{Maintenance Therapy}

The preference for ARI was increased and upgraded from first-line to the TOC for the combination with a MS to prevent manic episodes in the KMAP-BP 2018 when compared to the results from the KMAP-BP 2014. AAPs are preferred for preventing manic episodes in the KMAP-BP 2018; ARI, QUE and OLZ, for example, have proven efficacy. ${ }^{26)}$ However, the concerns for safety, such as metabolic syndrome, may favor the use of ARI, especially in combination with MSs, which could increase the risk of metabolic abnormalities in bipolar patients who need long-term maintenance treatment. ${ }^{54,55)}$

It is difficult to decide whether to maintain all medications or discontinue some in cases where the patient re- 
mitted after a combined treatment with AAPs, MSs or ADs. The general principle for maintenance is that medications found to be effective in the acute phase should be continued during the maintenance phase, except ADs. ${ }^{24)}$ However, in an actual clinical situation, many factors including intolerability, interactions among drugs, economic burden for the patient, and poor adherence due to complex dosing regimens necessitate discontinuing some medications. For example, the proportion of Korean experts who recommended continuing AAP was higher than those who recommended continuing a MS in the case of maintenance for a mixed state, and the representative case needs polypharmacy. This result could be interpreted as being that experts consider the role of AAPs more important than that of MSs during the maintenance phase. It is also noteworthy that the experts recommended discontinuing ADs 7 to 9 weeks after reaching remission. Because evidence-based guidelines or reviews have not reported when to discontinue ADs, due to limited data,24,25,56) this result could be informative for clinicians.

\section{Special Considerations}

Although ten anti-obesity agents, including metformin, topiramate, orlistat, bupropion, modafinil, zonisamide, amantadine, liraglutide, $\mathrm{H} 2$ receptor blockers, and sympathomimetic agents were included as choice options in the survey, interventions for diet and physical activity were not recommended as the TOC to manage a weight increase due to pharmacotherapy and no anti-obesity agents were recommended as the first-line. These results suggest that the experts did not consider these agents to be useful for treating bipolar patients who suffer from weight gain. KMAP-BP 2018 recommends switching to an AAP with a low risk for hyperprolactinemia as the first-line treatment option for patients with signs or symptoms of hyperprolactinemia, and a dose reduction of the current medication as the second-line option. These results are identical to the proposed algorithm for managing antipsychotic-induced hyperprolactinemia by Montejo et al. ${ }^{57)}$ who recommended switching antipsychotics as the first-line and dose adjustment as the second-line.

The KMAP-BP 2018 recommends reducing the dose of LMT when benign skin rashes develop, although treatment recommendations specify that LMT should be discontinued in patients who develop a rash, regardless of type and severity, unless the rash is clearly not drug related. ${ }^{58)}$ However, the incidence of serious rashes is rather low in patients with a mood disorder $(0.08 \%$ in adult patients with monotherapy and $0.13 \%$ in adult patients with adjunctive therapy) when compared to patients with epilepsy $(0.3 \%$ in adults patients with adjunctive therapy), ${ }^{59)}$ and there are several reports that LMT can be successfully reintroduced after emergence of a rash with a low dose and slow titration schedule. ${ }^{58)}$ Thus, the Korean experts recommended carefully continuing LMT at a reduced dose because of the risk of destabilizing symptoms and its unique role in the long-term prophylaxis of depressive episodes, although there have been no reliable data to support this recommendation until now.

The recommendation for geriatric patients with bipolar disorder in the KMAP-BP 2018 is comparable with those for adult patients, as recent reviews have suggested that medications efficacious in adult bipolar disorder will also be effective in older age patients with bipolar disorder, ${ }^{60,61)}$ with the exception that polypharmacy was generally graded lower, and ARI was more preferred for elderly than for adult patients. The devaluation of the combined treatment for older age patients with bipolar disorder is understandable in view of drug interactions due to frequent somatic comorbidities and altered drug metabolism. ${ }^{60)}$ Moreover, concerns about increasing the risk of comorbidities and complications with advancing age could raise the recommendation level of ARI in older age patients with bipolar disorder. ARI is considered relatively safe from common somatic comorbidities and complications in elderly patients including cardiac and metabolic adverse effects and sedation. ${ }^{62)}$

The preference for QUE in children decreased in this revision, as compared to the previous version. QUE was recommended as the first-line treatment for children with mania or depression in the KMAP-BP 2014, but it was downgraded to second-line for both mania and depression in the KMAP-BP 2018. Although RIS, ARI, and QUE carry US FDA approval and are recommended as first-line options to treat acute manic and mixed episodes in children in recent evidence-based guidelines, ${ }^{24,63)}$ QUE is not approved for use in pediatric patients in Korea. Only ARI is currently approved by the Korean FDA for treating bipolar manic and mixed episodes in children 10 to 17 years of age, and RIS is approved for aggressive children in the context of conduct disorder and disruptive behavior disorder. This drawback may restrict the use of QUE in 
children with bipolar disorder in Korea. However, QUE is recommended as the first-line treatment for mania in adolescents in the KMAP-BP 2018. This finding reflects the idea that the experts have a tendency to address children and adolescents with bipolar disorder differently.

A key limitation of this study is that it was based on consensus data from Korean experts rather than on experimental evidence. However, because the evidence is mainly derived from randomized controlled trials, generalizability could be limited, and the sample could be unrepresentative of real-world clinical practice. Accordingly, the KMAP-BP 2018 suggests a variety of treatment options based on expert recommendations that reflect the unique characteristics of the Korean healthcare environment, clinical experience, and experimental evidence. Additionally, because the KMAP-BP 2018 contains recommendations regarding clinical situations with limited evidence-based data, it will be helpful when dealing with diverse, challenging situations in real-world clinical practice. Another limitation of the present study is that the size of the review committee may be too small to reach a valid consensus. However, a sample of 127 psychiatrists may not be insufficient because there are only 3,254 psychiatrists in South Korea. Finally, this algorithm did not make any recommendations for novel pharmacological agents that have been supported by increasing amounts of evidence and have been recommended in recent foreign guidelines, such as lurasidone, asenapine, and caripra$z_{\text {zine }}{ }^{24,25}$ because they are not available in South Korea. Hence, this guideline could be limited in its use for clinicians in other countries, although it could be informative in some countries with restricted access to newer drugs.

To our knowledge, the KMAP-BP is the only set of treatment guidelines in Asia that has been updated and revised every 4 years since 2002. Thus, despite the limitations of this expert consensus, it is expected that the KMAP-BP 2018 will provide clinicians with a wealth of information regarding appropriate strategies for treating patients with bipolar disorder.

\section{- Acknowledgments}

The present manuscript is a secondary publication of our group's papers which were already published in the Korean language journal, 'Mood and Emotion'. Though we have already published the papers in Korea, we de- cided to present and share the results with the experts who speak English according to conditions for acceptable secondary publications as stated in Uniform Requirements for Manuscripts Submitted to Biomedical Journals by International Committee of Medical Journal Editors.

This study was supported by the Korean Society for Affective Disorders and the Korean College of Neuropsychopharmacology. This research did not receive any specific grant from funding agencies in the commercial sector.

\section{REFERENCES}

1. Parker GB, Graham RK, Tavella G. Is there consensus across international evidence-based guidelines for the management of bipolar disorder? Acta Psychiatr Scand 2017; 135:515-526.

2. Bahk WM, Shin YC, Jon DI, Yoon BH, Kim DJ, Ahn YM, et al. Korean Medication Algorithm for Bipolar Disorder (I). Korean J Psychopharmacol 2002;13:205-221.

3. Kim CH, Min KJ, Shin YC, Yun BH, Cho HS, Jon DI, et al. Feasibility of Korean Medication Algorithm for Bipolar Disorder (I): Global assessment. Korean I Psychopharmacol 2005; 16:225-233.

4. Shin YC, Bahk WM, Kim CH, Min KJ, Yun BH, Cho HS, et al. Feasibility of Korean Medication Algorithm for Bipolar Disorder (II): Choice of medications. Korean J Psychopharmacol 2005; 16:285-291.

5. Jon DI, Bahk WM, Shin YC, Kim CH, Min KJ, Yun BH, et al. Feasibility of Korean Medication Algorithm for Bipolar Disorder (III): Treatment response and tolerability. Korean J Psychopharmacol 2005; 16:292-300.

6. Seo JS, Bahk WM, Wang HR, Woo YS, Park YM, Jeong JH, et al. Korean Medication Algorithm for Depressive Disorders 2017: third revision. Clin Psychopharmacol Neurosci 2018;1 6:67-87.

7. Wang HR, Bahk WM, Seo JS, Woo YS, Park YM, Jeong JH, et al. Korean Medication Algorithm for Depressive Disorder: Comparisons with other treatment guidelines. Clin Psychopharmacol Neurosci 2017;15:199-209.

8. Jon DI, Bahk WM, Yoon BH, Shin YC, Cho HS, Lee E, et al. Revised Korean Medication Algorithm for Bipolar Disorder. World J Biol Psychiatry 2009; 10:846-855.

9. Shin YC, Min KJ, Yoon BH, Kim W, Jon DI, Seo JS, et al. Korean Medication Algorithm for Bipolar Disorder: second revision. Asia Pac Psychiatry 2013;5:301-308.

10. Woo YS, Lee JG, Jeong JH, Kim MD, Sohn I, Shim SH, et al. Korean Medication Algorithm Project for Bipolar Disorder: third revision. Neuropsychiatr Dis Treat 2015;11:493-506.

11. American Psychiatric Association; DSM-5 Task Force. Diagnostic and statistical manual of mental disorders, 5th ed: DSM-5. Arlington, VA:American Psychiatric Association;2013.

12. Sachs GS, Printz DJ, Kahn DA, Carpenter D, Docherty JP. The Expert Consensus Guideline Series: medication treatment of bipolar disorder 2000. Postgrad Med 2000;Spec No:1-104. 
13. Grunze H, Vieta E, Goodwin GM, Bowden C, Licht RW, Moller HJ, et al. The World Federation of Societies of Biological Psychiatry (WFSBP) guidelines for the biological treatment of bipolar disorders: update 2009 on the treatment of acute mania. World I Biol Psychiatry 2009;10:85-116.

14. Yatham LN, Kennedy SH, Parikh SV, Schaffer A, Beaulieu S, Alda M, et al. Canadian Network for Mood and Anxiety Treatments (CANMAT) and International Society for Bipolar Disorders (ISBD) collaborative update of CANMAT guidelines for the management of patients with bipolar disorder: update 2013. Bipolar Disord 2013;15:1-44.

15. Lin D, Mok H, Yatham LN. Polytherapy in bipolar disorder. CNS Drugs 2006;20:29-42.

16. Ketter TA. Monotherapy versus combined treatment with second-generation antipsychotics in bipolar disorder. I Clin Psychiatry 2008;69 Supp/ 5:9-15.

17. Bourin MS, Severus E, Schronen JP, Gass P, Szamosi J, Eriksson $\mathrm{H}$, et al. Lithium as add-on to quetiapine $X R$ in adult patients with acute mania: a 6-week, multicenter, double-blind, randomized, placebo-controlled study. Int J Bipolar Disord 2014;2:14.

18. Xu L, Lu Y, Yang Y, Zheng Y, Chen F, Lin Z. Olanzapine-va/proate combination versus olanzapine or valproate monotherapy in the treatment of bipolar I mania: a randomized controlled study in a Chinese population group. Neuropsychiatr Dis Treat 2015;11:1265-1271.

19. Ogawa Y, Tajika A, Takeshima N, Hayasaka Y, Furukawa TA. Mood stabilizers and antipsychotics for acute mania: a systematic review and meta-analysis of combination/augmentation therapy versus monotherapy. CNS Drugs 2014;28:989-1003.

20. Levin JB, Krivenko A, Howland M, Schlachet R, Sajatovic M. Medication adherence in patients with bipolar disorder: a comprehensive review. CNS Drugs 2016;30:819-835.

21. Leclerc E, Mansur RB, Brietzke E. Determinants of adherence to treatment in bipolar disorder: a comprehensive review. J Affect Disord 2013;149:247-252.

22. Galling B, Garcia MA, Osuchukwu U, Hagi K, Correll CU. Safety and tolerability of antipsychotic-mood stabilizer co-treatment in the management of acute bipolar disorder: results from a systematic review and exploratory meta-analysis. Expert Opin Drug Saf 2015;14:1181-1199.

23. Cipriani A, Barbui C, Salanti G, Rendell J, Brown R, Stockton $\mathrm{S}$, et al. Comparative efficacy and acceptability of antimanic drugs in acute mania: a multiple-treatments meta-analysis. Lancet 2011;378:1306-1315.

24. Yatham LN, Kennedy SH, Parikh SV, Schaffer A, Bond DJ, Frey BN, et al. Canadian Network for Mood and Anxiety Treatments (CANMAT) and International Society for Bipolar Disorders (ISBD) 2018 guidelines for the management of patients with bipolar disorder. Bipolar Disord 2018;20:97-170.

25. Fountoulakis KN, Grunze H, Vieta E, Young A, Yatham L, Blier P, et al. The International College of Neuro-Psychopharmacology (CINP) Treatment Guidelines for Bipolar Disorder in Adults
(CINP-BD-2017), Part 3: the clinical guidelines. Int J Neuropsychopharmacol 2017;20:180-195.

26. Lindström L, Lindström E, Nilsson M, Höistad M. Maintenance therapy with second generation antipsychotics for bipolar disorder - A systematic review and meta-analysis. J Affect Disord 2017;213:138-150.

27. Grunze H, Vieta E, Goodwin GM, Bowden C, Licht RW, Möller HJ, et al. The World Federation of Societies of Biological Psychiatry (WFSBP) guidelines for the biological treatment of bipolar disorders: update 2012 on the long-term treatment of bipolar disorder. World I Biol Psychiatry 2013;14:154-219.

28. Calabrese JR, Huffman RF, White RL, Edwards S, Thompson $\mathrm{TR}$, Ascher JA, et al. Lamotrigine in the acute treatment of bipolar depression: results of five double-blind, placebo-controlled clinical trials. Bipolar Disord 2008;10:323-333.

29. Unholzer S, Haen E. Retrospective analysis of therapeutic drug monitoring data for treatment of bipolar disorder with Lamotrigine. Pharmacopsychiatry 2015;48:296.

30. Solmi M, Veronese N, Zaninotto L, van der Loos ML, Gao K, Schaffer A, et al. Lamotrigine compared to placebo and other agents with antidepressant activity in patients with unipolar and bipolar depression: a comprehensive meta-analysis of efficacy and safety outcomes in short-term trials. CNS Spectr 2016;21:403-418.

31. National Collaborating Centre for Mental Health (UK). Bipolar disorder: the assessment and management of bipolar disorder in adults, children and young people in primary and secondary care, revised version. Leicester (UK):British Psychological Society;2014.

32. Goodwin GM, Haddad PM, Ferrier IN, Aronson JK, Barnes T, Cipriani A, et al. Evidence-based guidelines for treating bipolar disorder: Revised third edition recommendations from the British Association for Psychopharmacology. J Psychopharmacol 2016;30:495-553.

33. Vieta E. Antidepressants in bipolar I disorder: never as monotherapy. Am J Psychiatry 2014;171:1023-1026.

34. Shim IH, Woo YS, Wang HR, Bahk WM. Predictors of a shorter time to hospitalization in patients with bipolar disorder: Medication during the acute and maintenance phases and other clinical factors. Clin Psychopharmacol Neurosci 2017; 15:248-255.

35. Vázquez GH, Tondo L, Undurraga J, Baldessarini RJ. Overview of antidepressant treatment of bipolar depression. Int J Neuropsychopharmacol 2013;16:1673-1685.

36. Fountoulakis KN, Yatham L, Grunze H, Vieta E, Young A, Blier $\mathrm{P}$, et al. The International College of Neuro-Psychopharmacology (CINP) Treatment Guidelines for Bipolar Disorder in Adults (CINP-BD-2017), Part 2: Review, grading of the evidence, and a precise algorithm. Int I Neuropsychopharmacol 2017;20:121-179.

37. Cruz N, Sanchez-Moreno J, Torres F, Goikolea JM, Valentí M, Vieta E. Efficacy of modern antipsychotics in placebo-con- 
trolled trials in bipolar depression: a meta-analysis. Int J Neuropsychopharmacol 2010;13:5-14.

38. Sanford M, Keating GM. Quetiapine: a review of its use in the management of bipolar depression. CNS Drugs 2012;26:435460.

39. Yatham LN. A clinical review of Aripiprazole in bipolar depression and maintenance therapy of bipolar disorder. J Affect Disord 2011;128 Supp/ 1:S21-S28.

40. Thase ME, Bowden CL, Nashat M, Eudicone JM, Marcus R, McQuade RD, et al. Aripiprazole in bipolar depression: a pooled, post-hoc analysis by severity of core depressive symptoms. Int I Psychiatry Clin Pract 2012;16:121-131.

41. Fountoulakis KN, Vieta E, Schmidt F. Aripiprazole monotherapy in the treatment of bipolar disorder: a meta-analysis. J Affect Disord 2011;133:361-370.

42. Woo YS, Shim IH, Lee SY, Lee DB, Kim MD, Jung YE, et al. Dose trends of aripiprazole from 2004 to 2014 in psychiatric inpatients in Korea. Clin Psychopharmacol Neurosci 2017; 15:177-180.

43. Muneer A. Mixed states in bipolar disorder: etiology, pathogenesis and treatment. Chonnam Med I 2017;53:1-13.

44. Weisler RH, Hirschfeld R, Cutler AJ, Gazda T, Ketter TA, Keck $\mathrm{PE}$, et al. Extended-release carbamazepine capsules as monotherapy in bipolar disorder : pooled results from two randomised, double-blind, placebo-controlled trials. CNS Drugs 2006;20:219-231.

45. Stahl S, Lombardo I, Loebel A, Mandel FS. Efficacy of ziprasidone in dysphoric mania: pooled analysis of two double-blind studies. J Affect Disord 2010;122:39-45.

46. Swann AC, Bowden CL, Morris D, Calabrese JR, Petty F, Small J, et al. Depression during mania. Treatment response to lithium or divalproex. Arch Gen Psychiatry 1997;54:37-42.

47. Weisler RH, Kalali AH, Ketter TA; SPD417 Study Group. $A$ multicenter, randomized, double-blind, placebo-controlled trial of extended-release carbamazepine capsules as monotherapy for bipolar disorder patients with manic or mixed episodes. J Clin Psychiatry 2004;65:478-484.

48. Vieta E, Ramey T, Keller D, English PA, Loebel AD, Miceli J. Ziprasidone in the treatment of acute mania: a 12-week, placebo-controlled, haloperidol-referenced study. J Psychopharmacol 2010;24:547-558.

49. Zhang ZJ, Kang WH, Tan QR, Li Q, Gao CG, Zhang FG, et al. Adjunctive herbal medicine with carbamazepine for bipolar disorders: A double-blind, randomized, placebo-controlled study. J Psychiatr Res 2007;41:360-369.

50. Selle V, Schalkwijk S, Vázquez GH, Baldessarini RJ. Treatments for acute bipolar depression: meta-analyses of placebo-controlled, monotherapy trials of anticonvulsants, lithium and antipsychotics. Pharmacopsychiatry 2014;47: 43-52.
51. Judd LL, Akiskal HS, Schettler PJ, Endicott J, Maser J, Solomon DA, et al. The long-term natural history of the weekly symptomatic status of bipolar I disorder. Arch Gen Psychiatry 2002; 59:530-537.

52. Okuma T, Yamashita I, Takahashi R, Itoh H, Otsuki S, Watanabe $\mathrm{S}$, et al. Comparison of the antimanic efficacy of carbamazepine and lithium carbonate by double-blind controlled study. Pharmacopsychiatry 1990;23:143-150.

53. Small JG, Klapper MH, Milstein V, Kellams JJ, Miller MJ, Marhenke JD, et al. Carbamazepine compared with lithium in the treatment of mania. Arch Gen Psychiatry 1991;48: 915-921.

54. Rummel-Kluge C, Komossa K, Schwarz S, Hunger H, Schmid F, Lobos CA, et al. Head-to-head comparisons of metabolic side effects of second generation antipsychotics in the treatment of schizophrenia: a systematic review and meta-analysis. Schizophr Res 2010;123:225-233.

55. de Bartolomeis A, Perugi G. Combination of aripiprazole with mood stabilizers for the treatment of bipolar disorder: from acute mania to long-term maintenance. Expert Opin Pharmacother 2012;13:2027-2036.

56. Pacchiarotti I, Bond DJ, Baldessarini RJ, Nolen WA, Grunze $\mathrm{H}$, Licht RW, et al. The International Society for Bipolar Disorders (ISBD) task force report on antidepressant use in bipolar disorders. Am J Psychiatry 2013;170:1249-1262.

57. Montejo ÁL, Arango C, Bernardo M, Carrasco JL, CrespoFacorro B, Cruz JJ, et al. Multidisciplinary consensus on the therapeutic recommendations for iatrogenic hyperprolactinemia secondary to antipsychotics. Front Neuroendocrinol 2017:45:25-34.

58. Lorberg B, Youssef NA, Bhagwagar Z. Lamotrigine-associated rash: to rechallenge or not to rechallenge? Int I Neuropsychopharmacol 2009; 12:257-265.

59. Seo HJ, Chiesa A, Lee SJ, Patkar AA, Han C, Masand PS, et al. Safety and tolerability of lamotrigine: results from 12 placebo-controlled clinical trials and clinical implications. Clin Neuropharmacol 2011;34:39-47.

60. Dols A, Beekman A. Older age bipolar disorder. Psychiatr Clin North Am 2018;41:95-110.

61. Chen P, Dols A, Rej S, Sajatovic M. Update on the epidemio/ogy, diagnosis, and treatment of mania in older-age bipolar disorder. Curr Psychiatry Rep 2017;19:46.

62. Orsolini L, Tomasetti C, Valchera A, Vecchiotti R, Matarazzo I, Vellante F, et al. An update of safety of clinically used atypical antipsychotics. Expert Opin Drug Saf 2016;15:13291347.

63. Stepanova E, Findling RL. Psychopharmacology of bipolar disorders in children and adolescents. Pediatr Clin North Am 2017;64:1209-1222. 